REVISTA DE DERECHO UNED, NÚM. 23, 2018

\title{
DERECHO DE LA COMPETENCIA Y MERCADO DE SEGUROS: UN AÑO SIN REGLAMENTOS DE EXENCIÓN
}

\author{
COMPETITION LAW AND INSURANCE SECTOR: \\ ONE YEAR WITHOUT BLOCK EXEMPTION REGULATION
}

\author{
MANUEl I. MORÁN ARIAS \\ Doctorando en Derecho y Ciencias Sociales \\ Profesor-Tutor UNED. Abogado
}

Resumen: En marzo de 2017 expiró la vigencia del Reglamento 267/2010 de la Comisión, de 24 de marzo de 2010 relativo a la aplicación del artículo 101, apartado 3, del Tratado de Funcionamiento de la Unión Europea a determinadas categorías de acuerdos, decisiones y prácticas concertadas en el sector de los seguros. La Comisión acordó observar el impacto de la caducidad del reglamento durante los 12 meses posteriores para decidir sobre el futuro del sector asegurador. El presente trabajo analiza la regulación especial que durante años ha tenido el sector asegurador con los reglamentos de exención por categorías y los motivos que llevaron a la Comisión a no plantearse su renovación un año después de la caducidad del último.

Palabras clave: sector asegurador, Derecho de la competencia, reglamentos de exención.

Abstract: In March 2017 the validity of the Commission Regulation (EU) No 267/2010 of 24 March 2010 on the application of Article 101(3) of the Treaty on the Functioning of the European Union to certain categories of agreements, decisions and concerted practices in the insurance sector expired. The Commission agreed to observe the impact of the expiration of the Regulation during the following 12 months to decide on the future of the insurance sector. 
The present paper has analyzed the special regulation the insurance sector has had during years with the commissions regulations and the reasons that have led the Commission not to propose its renewal one year after its expiration.

Keywords: insurance sector, Competition Law, commission regulation.

Recepción original: 26/06/2018

Aceptación original: 21/09/2018

Sumario: I. Introducción.--II. El contrato de seguro y la normativa protectora de la libre competencia. 1. El Derecho anti-trust europeo: problemática inicial sobre su aplicación al sector asegurador. 2. Sentencia del Tribunal de Justicia de 27 de enero de 1987 («Verband der Sachversicherer») y la aplicación del Derecho antitrust al sector de los seguros. 3. Sentencia del Tribunal de Justicia (Sala Tercera) de 13 de julio de 2006 en el asunto Manfredi y la legitimación activa de cualquier persona para invocar sus derechos frente a los daños derivados por la infracción de las normas de defensa de la competencia.-III. Los reglamentos de exención por categorías en el sector asegurador. 1. Antiguos reglamentos A) Reglamento (CEE) 3932/1992, de 21 de diciembre, relativo a la aplicación del apartado 3 del artículo 85 del Tratado a determinadas categorías de acuerdos, decisiones y prácticas concertadas en el sector de los seguros. B) Reglamento (CE) N. ${ }^{\circ}$ 358/2003 de la Comisión de 27 de febrero relativo a la aplicación del apartado 3 del artículo 81 del Tratado a determinadas categorías de acuerdos, decisiones y prácticas concertadas en el sector de los seguros. 2. Último reglamento en vigor en el sector asegurador: Reglamento de UE 267/2010 de la Comisión relativo a la aplicación del artículo 101, apartado 3, del tratado de funcionamiento de la Unión europea a determinadas categorías de acuerdos, decisiones y prácticas concertadas en el sector de los seguros.-IV. Informe de la Comisión al Parlamento europeo y al Consejo sobre el funcionamiento del Reglamento (UE) n. ${ }^{\circ}$ 267/2010 de la Comisión relativo a la aplicación del artículo 101, apartado3, del Tratado de Funcionamiento de la Unión Europea a determinadas categorías de acuerdos, decisiones y prácticas concertadas en el sector asegurador. 1. Emisión del Informe. 2. Conclusiones acerca de la exención relativa al intercambio de información. 3. Conclusiones acerca de la exención relativa a la cobertura conjunta de ciertos tipos de riesgos. 4. Conclusiones respecto a la renovación del REC en materia de seguros.-V. Valoraciones finales y perspectivas futuras.-VI. Referencias bibliográficas. 


\section{INTRODUCCIÓN}

En virtud del artículo 101, apartado 3, del Tratado de Funcionamiento de la Unión Europea ${ }^{1}$, los acuerdos entre empresas que restrinjan la competencia en el sentido del artículo 101, apartado 1, del TFUE, podrán considerarse compatibles con el mercado interior si contribuyen a fomentar el progreso técnico o económico o a mejorar la producción o la distribución de los productos. Estas prácticas deben reservar al mismo tiempo a los usuarios una participación equitativa en el beneficio resultante, siempre y cuando las restricciones se limiten a lo estrictamente necesario - principio de proporcionalidad, y no eliminen la competencia por lo que se refiere a una parte sustancial del mercado de productos pertinente-.

En este contexto, escasos son los ámbitos en los que se prodigan más los acuerdos entre distintas empresas que en el asegurador, sector competitivo y oscilante como pocos ${ }^{2}$. Por ello, raros son los sectores en los que cobra tanta relevancia como en el asegurador la normativa protectora de la libre competencia ${ }^{3}$. Independientemente de la necesidad -indiscutible a nuestro modo de entender- de la existencia de esta colaboración en un ámbito tan particular como es el asegurador para su óptimo funcionamiento, dichas prácticas no pueden ser llevadas a cabo sin respetar la libertad concurrencial inherente al sistema de economía de mercado creado para la Unión Europea.

Hasta el pasado 31 de marzo de 2017, el sector asegurador gozaba de una regulación específica. Durante años la práctica aseguradora puso de manifiesto que las especialidades en este mercado concreto le hacían merecedor de esa regulación plasmada desde 1992 en los llamados reglamentos de exención por categorías ${ }^{4}$. El REC 267/2010 fue el último de este tipo de instrumentos por el cual la Comisión otorgó una especial regulación al sector asegurador.

1 En adelante TFUE.

${ }^{2}$ En este sentido VEIGA COPO, A. «El Derecho de Seguros en la Unión Europea», Revista Valenciana de Economía y Hacienda, n. ${ }^{\circ}$ 14, 2007, pg. 180; o CANTOS BAQUEDANO, F. y NAVEA GÓMEZ, G. en VV.AA. Tratado de Derecho de la competencia (BENEYTO, J.M.), Bosch, Tomo 3, 2005, pg. 1621.

${ }^{3}$ En palabras de VEIGA COPO, A. en «El Derecho de Seguros de la Unión Europea», op. cit., pg. 81 «En pocos sectores como el que nos atañe es más válida la idea de que la normativa comunitaria amparadora de la libre competencia, no sólo pretende mantener la competencia, la concurrencia en el mercado, sino que además trata de relanzar la consecución de una integración de los mercados en el tan deseado mercado único»:

${ }^{4}$ En adelante utilizaremos indistintamente el nombre completo o sus siglas REC.

(c) UNED. Revista de Derecho UNED, núm. 23, 2018 
Con motivo de su expiración, en el año 2016 la Comisión emitió un informe al Parlamento Europeo y al Consejo sobre el funcionamiento del REC seguros del año 2010, donde se plateaba la necesidad de renovar o no el mismo, como ya había sucedido anteriormente en dos ocasiones. El presente trabajo trata de analizar la regulación de la que el sector asegurador ha gozado durante años, así como los motivos esgrimidos por la Comisión para que esta situación de especialidad fuera mantenida en el tiempo un año después de la caducidad del REC 2010.

\section{EL CONTRATO DE SEGURO Y LA NORMATIVA PROTECTORA DE LA LIBRE COMPETENCIA}

\section{II.1. El Derecho anti-trust europeo: problemática inicial sobre su aplicación al sector asegurador}

En la segunda mitad del siglo pasado, el Derecho antitrust europeo quedaba sometido a lo dispuesto por el Reglamento N. ${ }^{\circ} 17$ para la aplicación de las reglas de la competencia ${ }^{5}$. Este texto, que mantuvo una vigencia de cuarenta años preveía un régimen en el que la prohibición recogida en el artículo 85.1 $\mathrm{TCE}^{6}$, entraba en juego de manera automática con reserva de autorización o de control previo. Los acuerdos colusorios llevados a cabo por las empresas que tuvieran intención de verse beneficiadas por la exención articulada en el precepto 85.3 TCE, debían ser expresamente notificados a la Comisión quien ostentaba la competencia exclusiva para la aplicación del citado artículo ${ }^{7}$.

Este sistema, que presentaba indudablemente ventajas para las empresas notificantes — siendo la seguridad jurídica otorgada la de mayor calado o envergadura- ocasionaba sin embargo considerables inconvenientes. Los operadores que acudían a la Comisión en solicitud de la exención, se enfrentaban a la siempre farragosa carga burocrática y a los importantes costes económicos que el proceso

${ }^{5}$ Reglamento n. ${ }^{\circ} 17$ del Consejo de la Comunidad Económica Europea de 6 de febrero de 1962, conocido como primer reglamento de aplicación de los artículos 85 y 86 del Tratado.

6 Tratado Constitutivo de la Unión Europea.

${ }^{7}$ Compartiendo con las autoridades de cada Estado miembro la competencia para la aplicación de los artículos 81.1 y 82 TCE, resolviéndose a favor de la Comisión cualquier conflicto cuando decidiera conocer de un determinado asunto que ya estuviera siendo conocido por la autoridad de algún Estado miembro, la cuál debía de abstenerse a favor de la Comisión. 
llevaba aparejado. Por su parte, la propia Comisión cargó con un enorme volumen de trabajo que irremediablemente retrasó la resolución de los expedientes sometidos a su consideración.

En este contexto, la aplicación de la normativa antitrust europea al sector asegurador no fue del todo pacífica, argumentándose inicialmente - con cierto éxito- su no aplicación a este mercado. Esta argumentación estaba amparada, al igual que se haría con el sector bancario, en razones de índole subjetiva pues las especialidades presentadas por el sector asegurador podrían hacerle valedor de un régimen distinto.

Hasta el año 1972, las peculiares características del sector asegurador $^{8}$ llevaron a muchos a entender de manera interesada y equívoca que las normas europeas en materia de competencia no eran aplicables a este sector. Tuvo que ser la Comisión, junto con el Segundo Informe sobre Política de la Competencia del citado año los que dejasen claro que el régimen de la competencia era $-\mathrm{y}$ siempre había sido - totalmente aplicable a los acuerdos y prácticas de las empresas aseguradoras. Con el apoyo de las clásicas sentencias del Tribunal de Justicia de las Comunidades en los asuntos «Van Ameyde ${ }^{9}$ y "Verband der Sachversicherer» ${ }^{10}$, se resolvió cualquier duda que pudiera subsistir sobre la posible aplicación de las normas de competencia al sector de los seguros.

${ }^{8}$ Particularidades recordadas por OLAVARRÍA IGLESIAS, J. en «La exención por categorías de la prohibición de prácticas colusorias en el sector de los seguros: el nuevo reglamento de la Comisión Europea», Revista Española de Seguros, Núm. 145, enero 2011, pg. 36 recogiendo ideas expuestas previamente por ALONSO SOTO, R. en "La aplicación de la normativa de la competencia al seguro», Revista Española de Seguros, Núm. 136, pg. 579, basadas en «el carácter especial del sector en atención a la existencia de un control estatal específico, que comportaba muchas veces la regulación de precios y condiciones comerciales, a la heterogeneidad y exhaustividad de las normas de ordenación y supervisión que lo regulan, a la primordial necesidad de tutela de un interés público consistente en garantizar la solvencia de las entidades aseguradoras e, incluso, a la consideración de que en este sector, debido a la inversión del ciclo productivo, el mecanismo de cálculo de los precios resulta diferente ya que los elementos de coste se sitúan en el futuro con respecto a las primas recibidas".

9 Sentencia del Tribunal de Justicia de 9 de Junio de 1977. Texto en inglés disponible en: http://eur-lex.europa.eu/LexUriServ/LexUriServ.do?uri=CELEX:61976CJ 0090:EN:HTML

10 Sentencia del Tribunal de Justicia de 27 de enero de 1987. Asunto 45/85. Disponible en http://eurlex.europa.eu/LexUriServ/LexUriServ.do?uri=CELEX:61985CJ0 045:ES:HTML 


\section{II.2. Sentencia del Tribunal de Justicia de 27 de enero de 1987 ( "Verband der Sachversicherer») y la aplicación del Derecho antitrust al sector de los seguros}

El 15 de febrero del año 1985, la asociación Verband der Sachversicherer.V. cuya finalidad era fundamentalmente la de representar, promover y proteger los intereses profesionales de los aseguradores que practicaban el seguro contra los riesgos industriales de incendio y de interrupción de explotación en el territorio de la República Federal Alemana, interpuso un recurso ante el Tribunal de Justicia, con el ánimo de obtener la anulación de la decisión de la Comisión de las Comunidades Europeas de 5 de diciembre 1984. La decisión impugnada hacía constar que la recomendación adoptada por la asociación Verband der Sachversicherer.V. en el año 1980, relativa a la estabilización y el saneamiento del sector asegurador contra los riesgos industriales de incendio y de interrupción de explotación constituía una infracción del artículo 85.1 del TCE.

La parte demandante, para fundamentar su recurso y con el apoyo de la Gesamtverband der deustschen Versicherungswirtschafte.V. - federación de las agrupaciones profesionales de empresarios de seguros en Alemania- que intervino en el litigio, en la primera de sus alegaciones afirmaba que el apartado 1 del artículo 85 no era aplicable —en ese momento y sin adaptaciones- al sector asegurador.

Sin negar que las normas sobre competencia contenidas en el Tratado fueran aplicables al sector de los seguros, la asociación Verband der Sachversicherer.V. sostenía haciendo referencia a un dictamen que oportunamente presentó al Tribunal de Justicia, que dichas normas sobre competencia no podrían ser aplicadas al sector asegurador hasta que el Consejo hubiera establecido las disposiciones de aplicación particulares para el sector que nos ocupa. Para reforzar sus argumentos, la parte demandante - con el apoyo de la parte coadyuvante- invocaba las peculiares características del sector de los seguros como elemento justificante para deducir que hasta que no se establecieran las deseadas disposiciones especiales en materia aseguradora, el artículo 85.1 TCE era inaplicable a este sector.

Por su parte, la Comisión alegaba la inexistencia de disposiciones especiales acomodables a los seguros reafirmándose en su posición de que las normas sobre competencia eran aplicables -en principio- a todos los sectores económicos y por lo tanto, aplicables también sin restricción al sector de los seguros. 
Para resolver el litigio, el Tribunal de Justicia en Sentencia de 27 de enero de 1987 falló en los siguientes términos:

"Cuando el Tratado ha pretendido excluir determinadas actividades de la aplicación de las normas sobre la competencia, ha establecido al efecto una excepción expresa. Tal es el caso de la producción y comercio de productos agrícolas en virtud del artículo 42 del Tratado. No existe en materia de seguros una disposición semejante que excluya la aplicación de las normas sobre la competencia o la subordine a una decisión del Consejo. Por consiguiente, el régimen comunitario sobre la competencia, tal como se contiene fundamentalmente en los artículos 85 y 86 del Tratado y en los preceptos del Reglamento $n .^{\circ} 17$, se aplica integralmente en el sector del seguro ${ }^{11}$.

Precisarlo así no significa en absoluto que el Derecho comunitario de la competencia no permita tener en cuenta las particularidades de determinados sectores de actividad económica. Incumbe en efecto a la Comisión, en el marco del apartado 3 del artículo 85 del Tratado, tener en cuenta la especial naturaleza de diferentes sectores económicos y las dificultades que les son propias.»

Con motivo de esta resolución, la Comisión recibió un aluvión de notificaciones por parte de las empresas aseguradoras -más de doscientas según puso de manifiesto en el XVII Informe de la Comisión sobre Política de la Competencia de 1989-que supusieron su colapso y redujeron la seguridad jurídica pretendida, pues en muchos casos la Comisión debía examinar supuestos prácticamente iguales ${ }^{12}$ y dictar decisiones de exención individual, corriendo el riesgo de que dos supuestos similares se resolvieran de manera distinta, sin restar importancia además a los largos y costosos procedimientos que la solicitud de una exención individual suponía.

Tratando de solucionar estos problemas, la Comisión propuso al Consejo la posibilidad de adoptar un reglamento de exención por categorías basándose en la experiencia acumulada en el tratamiento de casos individuales en el sector asegurador para la aplicación del artículo 85.3 TCE. Esta autorización se plasmó en el Reglamento (CEE) n. ${ }^{\circ} 1534 / 91$, de 31 de mayo, relativo a la aplicación del apartado 3 del artículo 85 del Tratado a determinadas categorías de acuerdos, decisiones y prácticas concertadas en el sector de los seguros, cuya adopción se justificaba y argumentaba en la idoneidad de la cooperación entre aseguradoras con objeto de garantizar el buen

11 El subrayado es nuestro.

12 Sirva como ejemplo los asuntos «TEKO» $\mathrm{y}$ «Concordatto Incendio» en los que la Comisión dictó dos decisiones de exención individuales para supuestos prácticamente idénticos. 
funcionamiento del mercado de seguros, buscando además la protección de los consumidores ${ }^{13}$.

\section{II.3. Sentencia del Tribunal de Justicia (Sala Tercera) de 13 de julio de 2006, en el asunto Manfredi y la legitimación activa de cualquier persona para invocar sus derechos frente a los daños derivados por la infracción de las normas de defensa de la competencia}

Un importante ejemplo de conducta prohibida por la normativa protectora de la libre competencia en el sector asegurador puede constatarse en la Sentencia del Tribunal de Justicia (Sala Tercera) de 13 de julio de 2006 en el asunto Manfredi ${ }^{14}$. En esta sentencia se analiza un interesante problema de aplicabilidad de la normativa de competencia europea al mercado de seguros italiano, a raíz de la vulneración del Derecho de competencia interno del citado país.

El asunto se sustenta en el marco de la interposición de varios recursos de indemnización ${ }^{15}$ por parte de algunos asegurados en Italia contra sus aseguradoras por el incremento llevado a cabo por dichas aseguradoras en las primas de seguro obligatorio de responsabilidad civil derivada de los siniestros causados por automóviles (RCA). Este incremento de precios, basado en un acuerdo llevado a cabo por las aseguradoras y declarado ilegal por la Autoridad nacional de defensa de la competencia italiana, era contrario al artículo 2

13 En su considerando 3. ${ }^{\circ}$ el Reglamento (CEE) n. ${ }^{\circ}$ 1534/91, de 31 de mayo establecía «que resulta deseable en cierta medida la cooperación entre empresas en el sector de seguros para garantizar el buen funcionamiento de este sector, al tiempo que puede salvaguardar los intereses de los consumidores».

${ }_{14}$ STJCE de 13.07.2006. en los asuntos acumulados C-295/04 a C-298/04. Para un análisis más detallado ver CARPAGNANO, M. «El prívate enforcement del derecho comunitario de la competencia en acción: Análisis crítico de la decisión del Tribunal de Justicia en los asuntos acumulados C295-298/04», Indret: Revista para el Análisis del Derecho, N. ${ }^{\circ} 1,2007$, pgs.1-21.

15 Dichas cuestiones se suscitaron en el marco de sendos recursos de indemnización interpuestos por el Sr. Manfredi contra Lloyd Adriatico Assicurazioni SpA, por el Sr. Cannito contra Fondiaria Sai SpA y por el Sr. Tricarico y la Sra. Murgolo, respectivamente, contra Assitalia SpA, con objeto de que se condenase a las citadas compañías aseguradoras a restituir los incrementos en las primas del seguro obligatorio de responsabilidad civil derivada de los siniestros causados por automóviles, embarcaciones y ciclomotores abonados a causa de los incrementos aplicados por dichas empresas en virtud de un acuerdo que fue declarado ilegal por la autoridad nacional de defensa de la competencia y del mercado. 
de la Ley de competencia $287 / 90^{16}$ y al Tribunal de Justicia Europeo se le planteó - junto con otras tres cuestiones prejudiciales- la necesidad de dilucidar sobre la aplicación del artículo 81 del TCE una vulneración del derecho nacional de la competencia. La especial relevancia que cobra este asunto, reside en la afirmación por parte del TJCE de «que un acuerdo o práctica concertada entre compañías aseguradoras consistente en un intercambio de información que permite un incremento de las primas del seguro de responsabilidad civil de automóviles no justificado por las condiciones del mercado, que vulnera las normas nacionales de defensa de la competencia, puede constituir también una infracción del artículo $81 C E^{17}$ si, habida cuenta de las características del mercado nacional en cuestión, existe un grado suficiente de probabilidad de que el acuerdo o práctica concertada examinado pueda ejercer una influencia directa o indirecta, real o potencial, en la venta de pólizas de este seguro en el Estado miembro de que se trate por parte de empresas establecidas en otros Estados miembros y de que esta influencia no sea insignificante» ${ }^{18}$.

De esta manera, la aplicación de la normativa nacional de protección de la competencia se llevará a cabo únicamente sobre los casos en los que pueda verse afectado el mercado nacional siempre y cuando esta actuación no pueda desembocar en una posible afectación del regular funcionamiento del mercado interior comunitario, ya que de ser así, será aplicable la normativa europea.

Esta sentencia, a su vez, se muestra de gran valor por todo lo referido en cuanto a la legitimación activa de cualquier persona para invocar sus derechos frente a los daños derivados por la infracción de las normas de defensa de la competencia. El Tribunal, afirmaba en el apartado 60 de la sentencia que «la plena eficacia del artículo 81 del Tratado y en particular su efecto útil se vería en entredicho si no existiera la posibilidad de que cualquier persona solicite la reparación del perjuicio que le haya irrogado un contrato o un comportamiento que pueda restringir o falsear el juego de la competencia».

${ }^{16}$ Legge 10 ottobre 1990, n.287, Norme para la tutela della concorrenza e del mercato, GURI n. ${ }^{\circ} 240$, de 13 de octubre de 1990.

17 El subrayado es nuestro.

18 Sentencia del Tribunal de Justicia (Sala Tercera) de 13 de julio de 2006, en los asuntos acumulados C-295/04 a C-298/04, apartado 52. 


\section{LOS REGLAMENTOS DE EXENCIÓN POR CATEGORÍAS EN EL SECTOR ASEGURADOR}

Como anunciábamos anteriormente ${ }^{19}$, en virtud del artículo 83 TCE, el Consejo aprobó el Reglamento 1534/91 que habilitaba a la Comisión a aprobar reglamentos que eximieran la prohibición general fijada por el artículo 81 TCE hasta seis categorías de acuerdos y prácticas concertadas entre aseguradoras.

Este texto se estructuró en torno a seis tipos o categorías de acuerdos entre empresas aseguradoras, que se describían en términos genéricos pero no se precisaban, siendo éstas:

a) Establecimiento en común de tarifas de primas de riesgo basadas en estadísticas de siniestralidad determinadas colectivamente o en el número de siniestros.

b) Establecimiento de condiciones tipo de las pólizas.

c) Cobertura en común de determinados tipos de riesgo.

d) Liquidación de siniestros.

e) Verificación y conformidad de los dispositivos de seguridad.

f) Registros de riesgos agravados y los correspondientes sistemas de información, siempre que la llevanza de dichos registros y la gestión de dicha información garanticen la confidencialidad.

Asimismo, para que las prácticas colusorias pretendidas por las empresas aseguradoras pudiesen exonerarse del cumplimiento de las reglas de competencia europea, se exigían requisitos adicionales importantes representados a través de las siguientes condiciones:

1. La obligación de que la duración del REC fuera limitada.

2. Que el REC pudiera ser susceptible de modificación o derogación si cambiaban las circunstancias que se tuvieron en cuenta para su aprobación.

3. Que la Comisión se reservara la facultad de retirar el beneficio de la exención por categorías en casos concretos en que se pruebe que en ella se amparan conductas que producen efectos incompatibles con las condiciones de exención previstas en el apartado 3 del hoy artículo 101 TFUE.

19 Vid. páginas 5 y 6 . 
4. Además de especiales requisitos procedimentales y de consulta pública previa a la aprobación del REC y de cualquiera de sus reformas.

Bajo la autorización otorgada por este texto se aprobó el primero de los reglamentos de exención por categorías destinado al sector asegurador ${ }^{20}$, que precedería a otros $\operatorname{dos}^{21}$ instrumentos que se han ocupado de parte de la problemática antitrust en el mercado de los seguros hasta la fecha presente. Las profundas modificaciones que sufrió el Derecho de la competencia en el año 2003, culminadas con la adopción del Reglamento 1/2003 del Consejo, de 16 de diciembre de 2002, relativo a la aplicación de las normas sobre competencia —que derogó el Reglamento núm. 17 de 1962-y que implantó un nuevo sistema de exceptuación legal con control a posteriori sustituyendo el hasta ese momento vigente sistema de prohibición con autorización o control previo, influyó, como no podía ser de otra manera, en los reglamentos de segunda y tercera generación que se han adoptado en la materia que nos ocupa.

Cabe destacar que a nivel nacional y de acuerdo con lo establecido por el artículo $1.4^{22}$ de la LDC, los reglamentos europeos de exención por categorías, aun en los casos en que los acuerdos y prácticas no afecten al comercio comunitario, serán directamente aplicables en España en relación con las prohibiciones del artículo 1 LDC.

${ }^{20}$ Reglamento (CEE) 3932/1992, de 21 de diciembre, relativo a la aplicación del apartado 3 del artículo 85 del Tratado a determinadas categorías de acuerdos, decisiones y prácticas concertadas en el sector de los seguros.

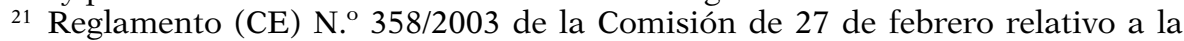
aplicación del apartado 3 del artículo 81 del Tratado a determinadas categorías de acuerdos, decisiones y prácticas concertadas en el sector de los seguros y Reglamento (UE) 267/2010 relativo a la aplicación del artículo 101, apartado 3, del tratado de funcionamiento de la Unión Europea a determinadas categorías de acuerdos, decisiones y prácticas concertadas en el sector de los seguros.

${ }^{22}$ La prohibición del apartado 1 no se aplicará a los acuerdos, decisiones, o recomendaciones colectivas, o prácticas concertadas o conscientemente paralelas que cumplan las disposiciones establecidas en los Reglamentos Comunitarios relativos a la aplicación del apartado 3 del artículo 81 del Tratado CE a determinadas categorías de acuerdos, decisiones de asociaciones de empresa y prácticas concertadas, incluso cuando las correspondientes conductas no puedan afectar al comercio entre los Estados miembros de la UE. 


\section{III.1. Antiguos reglamentos}

A) Reglamento (CEE) N. ${ }^{\circ}$ 3932/1992, de 21 de diciembre relativo a la aplicación del apartado 3 del artículo 85 del Tratado a determinadas categorías de acuerdos, decisiones y prácticas concertadas en el sector de los seguros

Haciendo uso de la habilitación otorgada por el Reglamento 1534/91, la Comisión, en este reglamento de primera generación sólo desarrolló un régimen completo de exención para cuatro de las seis categorías que inicialmente se habían previsto por el instrumento antes citado.

De esta manera, y justificándose en su falta de experiencia para establecer criterios claros de exención para su aplicación automática, la Comisión, dejaba fuera del ámbito de cobertura del reglamento a los acuerdos relativos a la liquidación de siniestros y a los que versaran sobre registros de riesgos agravados y los correspondientes sistemas de información.

El Reglamento (CEE) 3932/1992 estableció de esta manera que la exención automática recogida en su texto se aplicaría a los acuerdos, decisiones y prácticas concertadas relativas a:

a) la fijación en común de tarifas de primas de riesgo basadas en estadísticas colectivas o en el número de siniestros;

b) el establecimiento de condiciones tipo para los contratos de seguros;

c) la cobertura en común de determinados tipos de riesgos;

d) el establecimiento en común de normas para la verificación y aceptación de equipos de seguridad.

Parte de la doctrina ${ }^{23}$ opinaba que en consonancia con los reglamentos que le son coetáneos, el Reglamento (CEE) 3932/1992 puede ser considerado un texto muy formalista ${ }^{24}$ y poco flexible, que limi-

${ }^{23}$ En este sentido se manifiestan MARCOS FERNÁNDEZ, F. y SÁNCHEZ GRAELLS, A. en «Actividad aseguradora y defensa de la competencia: la exención antitrust del sector asegurador», Instituto de Ciencias del Seguro, Fundación Mapfre, 2011 , pg. 31.

${ }^{24}$ En palabras de MARCOS FERNÁNDEZ, F. y SÁNCHEZ GRAELLS, A. «poco orientado a criterios económicos y a los efectos derivadas de las prácticas objeto de la exención». Op. cit. pg. 31. 
taba los acuerdos de cooperación entre aseguradoras a los estrechos márgenes recogidos por su propio articulado.

Diez años después de su entrada en vigor ${ }^{25}$ y de acuerdo con su vigencia temporal limitada, el Reglamento 3932/1992 fue sustituido por el Reglamento (CE) 358/2003 de 27 de febrero.

B) Reglamento (CE) N. ${ }^{\circ}$ 358/2003 de la Comisión de 27 de febrero relativo a la aplicación del apartado 3 del artículo 81 del Tratado a determinadas categorías de acuerdos, decisiones y prácticas concertadas en el sector de los seguros

Con un período de vigencia de siete años a contar desde el día de su entrada en vigor ${ }^{26}$, el Reglamento (CE) N. ${ }^{0}$ 358/2003 renovó la exención para las cuatro categorías recogidas en el Reglamento precedente con el ánimo de seguir fomentando el adecuado funcionamiento de la actividad aseguradora.

Mediante su informe dirigido al Consejo y al Parlamento sobre la aplicación del Reglamento 3932/92, la Comisión se manifestaba satisfecha con el régimen de exención previsto para el sector asegurador y se mostraba conforme con su renovación introduciendo cambios poco significativos.

Estos cambios menores pasaban por actualizar el carácter formalista que presentaba el REC 92, fomentando una aproximación más económica a la evaluación de los pools de coaseguro o correaseguro $^{27}$, y la modificación de los requisitos para hacer aplicable la exención a los acuerdos de cobertura en común de determinados tipos de riesgos.

La estructura y condiciones de aplicabilidad de la exención para las restantes tres categorías se mantendría de manera invariable a la ya previstas en el REC 3932/92 aunque levemente modificadas ${ }^{28}$.

Con objeto de su caducidad el 31 de marzo de 2010, la Comisión Europea puso en marcha un proceso de propuesta de renova-

251 de abril del año 1993.

261 de Abril del año 2003.

27 En este sentido RYAN, S. "Commission publishes for comments a draft block exemption regulation for the insurance sector», Competition policy newsletter, n. ${ }^{\circ} 3$, 2002, pgs. 36.

${ }_{28}$ Las novedades que presentaba el REC2003 con respecto a su predecesor del año 1992 poseían fundamentalmente carácter técnico. De esta manera, el REC 2003 incorpora un mayor número de definiciones que las recogidas por el REC 1992. 
ción modificada del REC 2003 mediante consulta pública ${ }^{29}$, tras la cual emitió un Informe al Parlamento Europeo y al Consejo sobre el funcionamiento del Reglamento (CE) n. ${ }^{\circ} 358 / 2003^{30}$, trabajos que cristalizaron en la adopción del Reglamento (UE) 267/2010 relativo a la aplicación del artículo 101, apartado 3, del tratado de funcionamiento de la Unión Europea a determinadas categorías de acuerdos, decisiones y prácticas concertadas en el sector de los seguros.

\section{III.2. Último reglamento en vigor en el sector asegurador: Reglamento UE 267/2010, de la Comisión, relativo a la aplicación del artículo 101, apartado 3, del tratado de funcionamiento de la Unión europea a determinadas categorías de acuerdos, decisiones y prácticas concertadas en el sector de los seguros}

Con fecha 24 de marzo de 2010 se publicó la Comunicación de la Comisión relativa a la aplicación del artículo 101, apartado 3 del Tratado de Funcionamiento de la Unión Europea a determinadas categoría de acuerdos, decisiones y prácticas concertadas en el sector de los seguros, que acompañaba al Reglamento (UE) N. ${ }^{\circ}$ 267/2010.

En el apartado 4 de la Comunicación ${ }^{31}$, se establecía que dos de las cuatro excepciones recogidas por el Reglamento (CE) 358/2003 - los acuerdos sobre condiciones tipo de las pólizas y los dispositivos de seguridad- no serían renovadas por el REC (UE) 267/2010. Los motivos para su no renovación esgrimidos por la Comisión en este documento, se basaron en primer lugar en la no especificidad de estas formas de cooperación en el sector de los seguros. Se alegó por lo tanto, que su inclusión en un instrumento jurídico tan excepcional como son los reglamentos de exención por categorías, podría provocar una clara discriminación no justificada frente a otros sectores que no gozan de un REC.

En segundo término, y aunque reconocía la posibilidad de que estas formas de cooperación puedan originar beneficios para el mer-

${ }^{29}$ Lanzada el 17 de abril de 2008.

30 Comunicación de la Comisión de las Comunidades Europeas al Parlamento Europeo y al Consejo. Bruselas 24.03.2009. COM (2009) 138 final. Informe de la Comisión relativo a la aplicación del apartado 3 del artículo 81 del Tratado a determinadas categorías de acuerdos, decisiones y prácticas concertadas en el sector de los seguros.

Texto acompañado del Documento de trabajo del Informe de la Comisión relativo a la aplicación del Reglamento 358/2003.

31 Véase también el Considerando 3 del Reglamento (UE) 267/2003. 
cado de seguros y por lo tanto, para los consumidores, era posible también que su mantenimiento en un reglamento de exención por categorías provocara problemas de competencia, dictaminando que lo más conveniente era que quedasen sujetas a la autoevaluación por parte de las empresas.

Con su no inclusión en un REC específico era posible, como señalaba la Comisión, que los operadores en el sector de los seguros perdieran parte de la seguridad jurídica que les otorgaba el reglamento derogado, pero no sería menor que la de otros sectores que no cuentan con el beneficio que otorga un reglamento de exención.

El Reglamento UE 267/2010 relativo a la aplicación del artículo 101, apartado 3, del Tratado de Funcionamiento de la Unión Europea entró en vigor el 1 de abril del año 2010 y expiró el pasado 31 de marzo del año $2017^{32}$.

Este texto, introducía apreciables diferencias con respecto al régimen previsto por el reglamento que le precedía. Si bien como ya se ha señalado el REC 2010 sólo renovaba dos de las cuatro categorías que estaban exentas en el instrumento de 2003 — quedando pues sujetas a autoevaluación por parte de las empresas el resto de categorías-.

La primera de las exenciones renovada en el REC 2010, era la relativa a los acuerdos que perseguían como finalidad la elaboración de recopilaciones, tablas y estudios conjuntos ${ }^{33}$, que en consonancia con la terminología utilizada por el Reglamento habilitante 1534/1991 se correspondía con la exención inicialmente prevista para el establecimiento común de tarifas de primas de riesgo basadas en estadísticas de siniestralidad determinadas colectivamente o en el número de siniestros.

La renovación de esta exención por el Reglamento (CE) 267/2010 estaba motivada por las especiales características del sector asegurador o del producto que se ofrece - los seguros-, pues se estimaba que esta colaboración entre empresas de seguros o en el seno de asociaciones de empresas para recopilar información "permitía mejorar el conocimiento de los riesgos y facilita su evaluación por las distintas empresas» además de "facilitar, a su vez, la entrada en el mercado y beneficiar por lo tanto a los consumidores».

Para poder llevar a cabo un efectivo cálculo del riesgo, razonaba la Comisión, se precisa contar necesariamente con datos estadísticos

32 Capítulo IV. Disposiciones finales. Artículo 9.

${ }^{33}$ Capítulo II, artículo 2 Reglamento (UE) 267/2010. 
sobre el pasado, números que únicamente pueden obtenerse a través de una colaboración considerada totalmente específica del sector de los seguros que hacía necesaria el mantenimiento de una exención automática en un instrumento tan específico como es un REC.

A su vez, recopilar y elaborar esta información según se expuso en ese momento, suponía una tarea con un coste tan cuantioso, que sólo el reconocimiento de esta exención por medio de un reglamento permitía, razonó la Comisión, a las empresas de pequeño y mediano tamaño el acceso sin mayores dificultades a la actividad aseguradora, así como a las aseguradoras que ya instaladas en determinado mercado geográfico o vinculados a un ramo concreto, tuvieran intención de ampliar su actividad a otros mercados o riesgos distintos. Todo este planteamiento se completaba con la obligación que imponía el reglamento de dar acceso a la información recopilada, de modo no discriminatorio a otras compañías de seguros.

Específicamente, el reglamento señalaba que la exención era aplicable siempre que las recopilaciones o tablas:

«a) se basasen en una recopilación de datos que abarquen varios años-riesgo elegidos como período de observación y se refiriesen a riesgos idénticos o comparables en un número suficiente para constituir una base que pueda ser objeto de tratamiento estadístico y permitiera cifrar, entre otras cosas, sobre lo siguiente:

i) el número de siniestros durante dicho período,

ii) el número de riesgos individuales asegurados en cada añoriesgo del período de observación elegido,

iii) los importes totales pagados o por pagar en concepto de siniestros acaecidos durante dicho período,

iv) el importe total de capital asegurado en cada año-riesgo durante el período de observación elegido;

b) incluyan un desglose de las estadísticas disponibles tan detallado como resulte adecuado a efectos actuariales;

c) no contengan en forma alguna, recargos de seguridad, ingresos procedentes de reservas, costes administrativos o comerciales o contribuciones fiscales o parafiscales, y no tengan en cuenta los ingresos obtenidos de las inversiones ni los beneficios anticipados.

2. Las exenciones previstas en el artículo 2 se aplicaban siempre que las recopilaciones, las tablas o los resultados de los estudios:

a) no revelen la identidad de las empresas de seguros afectadas o de alguna parte asegurada;

b) una vez recopilados y difundidos, contengan una declaración de que no son vinculantes; 
c) no contengan ninguna indicación sobre el nivel de las primas comerciales;

d) se pongan a disposición, en condiciones razonables, asequibles y no discriminatorias, de toda empresa de seguros que pida una copia de ellos, incluidas las que no operen en el mercado geográfico o de productos al que se refieran dichas recopilaciones, tablas o resultados de estudios.»

En cuanto a la segunda exención, en consonancia con lo ya reconocido por los reglamentos de exención de los años 1992 y 2003, el REC 2010 renovó la exención para los «acuerdos celebrados entre dos o más empresas del sector de seguros relativos a la constitución y el funcionamiento de agrupaciones de empresas de seguros o de empresas de seguros y empresas de reaseguros para la cobertura conjunta de una categoría específica de riesgos en forma de coaseguro o correaseguro ${ }^{34}$.

A pesar de que el REC 2010 mantenía el carácter continuista ya señalado con respecto a estas prácticas colusorias, como ya lo hacía su predecesor, el nuevo REC introducía cambios reseñables —calificados de sustantivos en la Comunicación por la Comisión- que modificaban la exención configurándola en los siguientes términos:

- Se preveía la obligatoriedad de que las empresas agrupadas, desplegasen su actividad en régimen de coaseguro o correaseguro, resultando fundamental la vocación de permanencia o institucionalización de la agrupación, dado que cualquier tipo de acuerdo no iba a quedar cubierto por el reglamento.

- El régimen al que eran sometidas las agrupaciones destinadas a dar cobertura exclusivamente a riesgos nuevos, sería distinto del que recibían aquéllas que iban dirigidas a cubrir riegos distintos a los anteriormente citados.

- El criterio de la no superación de determinados umbrales de cuota de mercado era relevante para determinar la aplicabilidad de la exención a las agrupaciones destinadas a cubrir riesgos no considerados nuevos, o para los que teniendo tal consideración, hubiera sido superado el lapso de tiempo previsto por el reglamento.

La Comisión señaló que la cooperación para dar cobertura «a ciertos tipos de riesgos - tales como los de índole nuclear, terrorista o medioambiental-, que las compañías de seguros individuales no

${ }^{34}$ Capítulo III. Artículo 5 Reglamento (UE) 267/2010. 
pueden o no quieren asegurar solas en su totalidad, es crucial para asegurarse de que se puedan cubrir todos los riesgos de este tipo ${ }^{35}$.

Esta especificidad que presenta el sector de los seguros, le hacía en ese momento diferente de otros sectores y justificaba el mantenimiento de la exención para este tipo de acuerdos de cooperación en un REC. De otra manera, la cobertura ofrecida por las aseguradoras a determinados tipos de riesgo - por lo cuantioso de su aseguramiento o lo novedoso de su naturaleza- podía ser insuficiente, razón por la que se optó por considerar que este tipo de agrupaciones no serían contrarias a lo establecido en el artículo 101.1 TFUE ya que no suponían con carácter general una verdadera restricción a la competencia, sino la única manera eficaz de cubrir los riesgos citados.

Esta regulación que se mantuvo vigente hasta marzo del año pasado requería, pasados seis años de su entrada en vigor con arreglo al Artículo 8 del Reglamento de habilitación, de un informe de la Comisión sobre el funcionamiento del REC y sus perspectivas de futuro.

La renovación, renovación parcial o su no renovación dependería de dicho informe que fue emitido por la Comisión el 17 de marzo de $2016^{36}$.

IV. INFORME DE LA COMISIÓN AL PARLAMENTO EUROPEO

Y AL CONSEJO SOBRE EL FUNCIONAMIENTO DEL

REGLAMENTO (UE) N. . 267/2010, DE LA COMISIÓN, RELATIVO A LA APLICACIÓN DEL ARTÍCULO 101, APARTADO 3, DEL TRATADO DE FUNCIONAMIENTO DE LA UNIÓN EUROPEA A DETERMINADAS CATEGORÍAS DE ACUERDOS, DECISIONES Y PRÁCTICAS CONCERTADAS EN EL SECTOR ASEGURADOR

\section{IV.1. Emisión del Informe}

En febrero de 2014 se inició una revisión formal del uso y funcionamiento del REC 267/2010. En su considerando séptimo de su

35 Comunicación de la Comisión relativa a la aplicación del artículo 101, apartado 3, del Tratado de Funcionamiento de la Unión Europea a determinadas categorías de acuerdos, decisiones y prácticas concertadas en el sector de los seguros. Apartado 12.

${ }^{36}$ En: http://eur-lex.europa.eu/legal-content/ES/TXT/?uri=CELEX\% 3A52016DC0153 
informe, la Comisión recuerda que durante el segundo semestre del 2014 se realizaron consultas y reuniones con los entes interesados ${ }^{37}$ - autoridades nacionales en materia de la competencia y asociaciones y agrupaciones de aseguradoras-con el ánimo de recabar pruebas para responder a las siguientes preguntas:

- ¿Se sigue caracterizando el sector de los seguros por poseer unos rasgos específicos que presentan una necesidad mayor de cooperación en los ámbitos a los que se aplican el resto de exenciones del REC?

- En tal caso, ¿sigue siendo necesario un instrumento excepcional como el REC para proteger esa mayor necesidad de cooperación? ${ }^{38}$

A lo largo de documento, la Comisión se detiene a analizar las dos excepciones que el REC 2010 renovaba, razonando sobre su funcionamiento y obteniendo una serie de conclusiones que analizaremos a continuación.

\section{IV.2. Conclusiones acerca de la exención relativa al intercambio de información}

Con respecto al intercambio de información que hasta el año 2017 era permitido bajo las condiciones del REC, la Comisión define a los seguros como un producto que cubre riesgos futuros cuyo coste se ignora al suscribirse el contrato de seguro. Resulta según sus palabras, crucial disponer de información estadística anterior que sea adecuada y precisa sobre el coste real de las clases de riesgo para las operaciones en los distintos segmentos de la actividad aseguradora. Mediante el intercambio de información en forma de recopilaciones, tablas y estudios conjuntos, los aseguradores pueden cotejar estadísticas más fiables y llegar a una mejor comprensión de los riesgos asegurados. Esto permitirá evaluar los riesgos de manera más fiable y, como consecuencia, reducir los márgenes comerciales

37 Este proceso de consulta que se inició en el año 2014, no se realizó exactamente bajo las prácticas llevadas a cabo por la Comisión en los últimos años. Cabe recordar que en mayo de 2015, la Comisión Europea adoptó un nuevo enfoque para «Legislar mejor», encaminado a realizar consultas más sistemáticas y transparentes a las partes interesadas, mejorar las evaluaciones de impacto y extraer lecciones de la experiencia acumulada. Aún así en su considerando 12, la Comisión advertía que el proceso de revisión fue realizado de un modo abierto y transparente, respetando los elementos clave de la evaluación.

38 Vid. Considerando 10 del Informe. 
y en última instancia los precios abonados por los consumidores. El intercambio de datos estadísticos anteriores conduce al funcionamiento eficiente del sector de los seguros y al realizar estos estudios de modo conjunto, los aseguradores toman mayor conciencia de la probable evolución futura de los riesgos en cuestión ${ }^{39}$.

Aun reconociendo esta especialidad, la Comisión razona que no es algo exclusivo del sector asegurador y por lo tanto se plantea la necesidad de ver justificado el mantenimiento de una regulación diferenciada en este mercado.

Con objeto de plantear la no renovación del REC en el sector asegurador, la Comisión invitó en la consulta pública a que las partes interesadas ofrecieran una descripción pormenorizada de las posibles modificaciones concretas de su conducta comercial y cuantificaran, o al menos clasificaran los costes adicionales en que incurrirían como consecuencia de ello, así como su repercusión en las primas $^{40}$. Las respuestas de las partes interesadas favorables al mantenimiento de esta excepción en un nuevo reglamento de exención por categorías, no pudieron ofrecer en ningún caso argumentos de peso para su mantenimiento. Mediante argumentos generales, los interesados hicieron hincapié en los efectos negativos que produciría la desaparición de esta cooperación, que derivaría según los mismos principalmente en una mayor «inseguridad jurídica» no apoyada por los datos y estadísticas que la Comisión requería.

Según los encuestados, la percepción de esta inseguridad jurídica tendría varios efectos negativos, incluidos unos costes de conformidad más elevados y, posiblemente, una menor colaboración. Igualmente, los interesados sugirieron que los titulares de más tamaño, que puedan no necesitar datos estadísticos adicionales para evaluar los riesgos con exactitud, podrían no sentirse inclinados a compartir esta información con aseguradores de menor tamaño y posibles nuevos operadores, quienes se enfrentan a mayores dificultades a la hora de calcular los riesgos, habida cuenta de su presencia limitada o nula en el mercado, lo que disminuiría la competencia y perjudicaría los intereses de los consumidores ${ }^{41}$. El principal argumento esgrimido para el mantenimiento del REC por los operadores consultados, estaba basado en la insuficiente justificación de que la información estadística acumulada y anónima sobre los riesgos se divulgue en condiciones razonables, asequibles y no discriminatorias

39 Vid. Considerando 15 del Informe.

40 Vid. Considerando 23 del Informe.

${ }^{41}$ Vid. Considerando 25 del Informe. 
garantiza unas condiciones de competencia equitativas entre los aseguradores de mayor y menor tamaño, y facilita la entrada en el mercado.

En la ronda de consultas, los interesados también afirmaron que la menor divulgación de información relativa a los riesgos podría mermar la confianza en el cálculo obligatorio por parte de los aseguradores de sus pasivos basándose en la «mejor estimación», y podría dar lugar a más disposiciones al objeto de constituir reservas suficientes para inseguridades y pasivos contingentes.

Ante esta falta de argumentos concluyentes, la Comisión manifestó que existen orientaciones adecuadas para garantizar los beneficios del intercambio de información recogidas en las Directrices horizontales ${ }^{42}$ adoptadas tras la entrada en vigor del REC 267/2010. Esta Directrices son plenamente aplicables al sector asegurador y por lo tanto desde ese momento, era cuestionable el mantenimiento de un instrumento diferenciador - como el REC en el sector asegurador-cuando existen elementos alternativos de orientación.

Las Directrices reconocen de forma expresa en su apartado 89 que los intercambios de datos verdaderamente agregados, es decir, aquellos que dificultan suficientemente el reconocimiento de la información individualizada de cada empresa, tienen muchas menos probabilidades de producir efectos restrictivos de la competencia que los intercambios de datos individuales de cada empresa. Igualmente, en el mismo apartado se reconoce que la recogida y publicación de datos de mercado agregados obtenidos por una organización comercial o por una empresa de información sobre el mercado pueden beneficiar tanto a los proveedores como a los consumidores al darles una idea más fehaciente de la situación económica del sector. Esa recogida y publicación de datos puede permitir a quienes participan en el mercado una mejor toma de decisiones individuales para adaptar más eficientemente su estrategia a las condiciones del mercado. Concluía la Comisión que a menos de que ocurra en un oligopolio muy restringido, el intercambio de datos agregados es poco probable que tenga efectos restrictivos de la competencia ${ }^{43}$.

En su apartado 97, las Directrices también reconocen que el intercambio de datos sobre clientes entre empresas que operan en mercados con información asimétrica sobre aquellos puede dar lugar a eficiencias, permitiendo detectar a los consumidores que supo-

42 Directrices sobre la aplicabilidad del artículo 101 del Tratado de Funcionamiento de la Unión Europea a los acuerdos de cooperación horizontal.

${ }^{43} \mathrm{Vid}$. Considerando 29 del Informe. 
nen un menor riesgo y deben beneficiarse de precios más bajos. El intercambio de información también puede reducir la cautividad de clientes, induciendo así una mayor competencia.

Argumentando lo anteriormente expuesto, la Comisión razona que en caso de que se eliminara el REC, ya se habrían establecido directrices equivalentes de la Comisión para ayudar a los aseguradores a autoevaluar la admisibilidad de su colaboración ${ }^{44}$ y por lo tanto el mantenimiento de esta especialidad en un Reglamento de Exención por categorías era cuestionable.

\section{IV.3. Conclusiones acerca de la exención relativa a la cobertura conjunta de ciertos tipos de riesgos}

Con respecto a la Cobertura conjunta de ciertos tipos de riesgos (agrupaciones) permitida por el REC 267, en su considerando número 19 del Informe, la Comisión reconocía que los aseguradores a menudo tienen que colaborar para la cobertura de algunos riesgos no convencionales de gran magnitud. En los casos en los que la naturaleza del riesgo es tal que ningún asegurador puede ofrecer la capacidad necesaria, el coaseguro o el correaseguro permite a los aseguradores cubrir un número suficiente de riesgos.

Teniendo esto presente, de la información manejada por la Comisión se desprendía que en la última década el mercado de seguros ha desarrollado formas de cooperación más competitivas, como el coaseguro o correaseguro a través de corredores o propuestas de distribución del riesgo. Estos métodos de cooperación se han mostrado como una alternativa viable a los excepciones protegidas por el hoy caducado REC de 2010, que no podía mantener según la Comisión una especialidad reguladora que no era únicamente propia de este sector, ya que, por ejemplo la colaboración en proyectos específicos de gran magnitud con el fin de repartir costes y riesgos, se aplica también a los grandes proyectos de construcción.

Una vez más, la consulta realizada a las partes interesadas no pudo arrojar datos y pruebas concluyentes diferentes a la alegación de una posible inseguridad jurídica no justificada. Esta argumentación estaba basada únicamente en la afirmación general de que permitir que la exención de las agrupaciones caduque daría lugar a una reducción de la seguridad jurídica en cuanto a la admisibilidad de la colaboración en forma de agrupaciones, lo que, a su vez, aumenta-

${ }^{44}$ Vid. Considerando 31 del Informe. 
ría los costes de conformidad para los aseguradores y provocaría un exceso de precaución en lo que respecta a la celebración de algunos contratos de agrupación. Se aduce que ello podría dar lugar a que los aseguradores cobren primas más elevadas y, en algunos casos, incluso a la disolución de agrupaciones existentes, en particular en el ámbito de los riesgos agravados y de catástrofe, lo que podría derivar en la suspensión de algunos productos de coaseguro, en detrimento de los consumidores ${ }^{45}$.

La falta de argumentos suficientes para justificar la renovación del REC en el sector asegurador, se hizo patente a lo largo del pormenorizado estudio ${ }^{46}$ que la Comisión llevó a cabo antes del lanzamiento de la consulta pública sobre las agrupaciones de coaseguro y correaseguro y acuerdos ad hoc de coaseguro y correaseguro en el mercado de suscripción basado en entrevistas llevadas a cabo en toda la Europa de los Veintisiete. Únicamente se identificaron 46 agrupaciones activas en toda la UE basándose en la definición del $\mathrm{REC}^{47}$ —número significativamente inferior al esperado inicialmente-.El estudio concluía que muchas agrupaciones se constituyen para cubrir riesgos catastróficos (nuclear, medioambiental, terrorismo) pero no son la única alternativa a tales riesgos, habida cuenta de que existen mercados de seguros y otros mecanismos, como aseguradores con garantía estatal, que también cubren algunos de estos riesgos. Junto a estas agrupaciones existen otros acuerdos entre aseguradores para hacer frente a riesgos que el mercado de seguros no desea, o para aprovechar un nicho de mercado donde los aseguradores se unen para proporcionar capacidad en el mercado de suscripción.

Con respecto al uso de la exención de las agrupaciones, los datos arrojados por el estudio resultaron igualmente decepcionantes. Algunas de estas agrupaciones no habían llevado a cabo ninguna autoevaluación completa al considerarse exentas de ello por cubrir riesgos nuevos o porque confiaban en que su cuota de mercado era inferior al umbral del $20 \%$. En general, el conocimiento del REC de seguros parecía contradictorio, pese a que aquellas agrupaciones que habían reevaluado su posición desde la publicación del nuevo REC no informaron de ningún cambio en su situación de conformidad. Anali-

45 Vid. Considerando 34 del Informe.

${ }^{46}$ Study on co(re)insurance pools and on ad hoc co(re)insurance agreements on the subscription market. - Estudio sobre agrupaciones de coaseguro y correaseguro y acuerdos ad hoc de coaseguro y correaseguro en el mercado de suscripción. Texto en inglés disponible en: http://ec.europa.eu/competition/sectors/financial_services/ KD0414707ENN.pdf

47 Vid. Considerando 37 del Informe. 
zando su funcionamiento, el estudio concluyó que las agrupaciones actúan de manera heterogénea y cada agrupación precisa una evaluación de sus méritos individuales ${ }^{48}$.

Los resultados del estudio, confirmaron las siguientes constataciones:

a) en el mercado de coaseguro y correaseguro ya existen formas de colaboración que difieren de las agrupaciones pero que desempeñan una función similar;

b) tiende a producirse una falta de claridad en el mercado en cuanto a cuáles de estas formas constituyen agrupaciones de coaseguro o correaseguro en el sentido del REC y que podrían, por tanto, beneficiarse de la exención prevista para las agrupaciones;

c) la definición geográfica y de producto de los mercados en los que se encuentran activas las agrupaciones, y la definición de riesgos nuevos con arreglo al REC, pueden no quedar claros para todos los participantes del mercado; y

d) pese a que los participantes en el mercado piensan que las agrupaciones aún son necesarias para determinados riesgos, reconocieron que el mercado tal vez esté avanzando hacia soluciones de coaseguro y correaseguro más flexibles y que favorezcan la competencia. ${ }^{49}$

El artículo 5 del REC estipulaba que quedaban exentos los acuerdos de cooperación entre empresas de seguros relativos a la constitución y funcionamiento de agrupaciones. A lo largo de los años, la práctica decisoria de la Comisión ${ }^{50}$ ha distinguido siempre entre «constitución» y "funcionamiento». En relación con la primera, la Comisión considera que el mero hecho de que una agrupación

${ }^{48}$ La revisión reveló una significativa y creciente tendencia del mercado a alejarse de las agrupaciones institucionalizadas constituidas a iniciativa de los propios aseguradores y a dirigirse hacia formas de colaboración entre aseguradores o reaseguradores más favorables a la competencia. Vid. Considerando 43 del Informe.

${ }^{49}$ Las conclusiones llevadas a cabo por el Estudio son reproducidas por la Comisión en el Considerando 41 del Informe.

${ }^{50}$ Entre otros ejemplos: Decisión P\&I Clubs de 1985 (asuntos 30.373 y 37.143); Decisión Assurpol; Decisión TEKO; Decisión Lloyd's/ILU; cuatro cartas de compatibilidad para las agrupaciones nucleares, Svenska Atomförsäkringspoolen (asunto COMP/37.363), Pool Italiano Rischi Atomici (asunto COMP/34.985), y Aseguradores Riesgos Nucleares (asunto COMP/34.558); véase el XXXI Informe sobre la política de competencia 2001, apartado 203, y Deutsche Kernreaktorversicherungsgemeinschaft (DKVG) (asunto COMP/36.053), 
pueda resultar necesaria no constituye razón suficiente para pensar que no distorsiona la competencia. Con respecto al funcionamiento de la agrupación, en varios asuntos ${ }^{51}$ la Comisión considera que una agrupación no puede ser compatible en términos generales si su viabilidad pudiera garantizarse con arreglo a condiciones de funcionamiento menos restrictivas.

Tras una evaluación en profundidad de la información recabada en el transcurso de la revisión, la Comisión consideró que la exención de las agrupaciones puede no ajustarse a las condiciones estrictas para la creación de un instrumento excepcional como un REC. Las respuestas recibidas durante la revisión de la situación competitiva fueron contradictorias, la información obtenida de la revisión constató que la exención de agrupaciones parecía tener una relevancia de uso marginal, concluyendo la Comisión que un REC para una categoría determinada de acuerdo solo se justifica cuando se puede presumir con certeza suficiente que la categoría produce realmente beneficios que se comparten de forma equitativa con los consumidores.

En consecuencia, finalizaba la Comisión que en las actuales circunstancias de mercado y a la luz de la información disponible sobre el uso y funcionamiento del REC, ya no es posible suponer que los acuerdos de agrupaciones restrictivos que contempla el REC cumplan las cuatro condiciones de compatibilidad estipuladas en el artículo 101, apartado 3, del TFUE ${ }^{52}$.

\section{IV.4. Conclusiones respecto a la renovación del REC en materia de seguros}

A pesar de que la Comisión en el año 2016 destacaba que sus conclusiones y constataciones tenían un carácter preliminar, supeditadas a la evaluación de las observaciones y conversaciones con las partes interesadas en el sector que estaban siendo llevadas a cabo por la Comisión, todo parecía indicar que en el Informe de principios de 2017 — como así sucedió- las especialidades en el sector asegurador no serían mantenidas, reservándose la Comisión la posibilidad de decidir o adoptar orientaciones específicas sobre los principios de autoevaluación aplicables por las entidades que ya no se beneficien de un REC.

51 Sirva como ejemplo Assurpol o P\&I Clubs.

52 Vid. Considerando 47 del Informe. 
La Comisión en el apartado final de su informe razonaba que, pese a que existen indicios de una mayor necesidad de colaboración en el sector de los seguros en cuanto a la recopilación y distribución de estimaciones, tablas y estudios conjuntos y el coaseguro o correaseguro de tipos concretos de riesgo, ya no parecen cumplirse las estrictas condiciones para la creación de un REC específico del sector en lo que se refiere a estas categorías de acuerdos ${ }^{53}$.

A escasos meses de la caducidad del reglamento de exención del año 2010, se publicó el Informe de evaluación de impacto de la Comisión ${ }^{54}$ en el que se decidiría, finalmente, permitir la caducidad del REC seguros en la fecha prevista y observar el sector asegurador durante 12 meses para decidir sobre su futuro.

\section{VALORACIONES FINALES Y PERSPECTIVAS FUTURAS}

Las reticencias que siempre ha mostrado la Comisión Europea para reconocer la existencia de sectores que pueden ser considerados como especiales ${ }^{55}$, se han visto durante años matizadas por el mantenimiento de un reglamento de exención por categorías en el sector asegurador. Esta normativa destinada exclusivamente al ámbito de los seguros, no hacía sino confirmar las particulares características del sector que nos ocupa.

Aun así, no podemos olvidar que la progresiva pérdida de privilegios - entendidos como elementos diferenciadores de otros sectores- del mercado asegurador manifestada a través de la reducción de exenciones reconocidas a lo largo de los años, hacía pensar, como ya lo vaticinaba algunos autores ${ }^{56}$, que este tratamiento diferenciado posiblemente llegaría su fin en un futuro no muy lejano. Esto se hizo patente en el momento en que la Comisión decidió permitir la expiración del REC 267/2010 en marzo de 2017. En este momento, no parecen cumplirse las estrictas condiciones para la creación de un REC específico para el sector asegurador, estando aún por ver si la

53 Vid. Considerando 50.

${ }^{54}$ Comission Staff Working Document. Executive summary of the Impact Assesment disponible: http://ec.europa.eu/competition/sectors/financial_services/iber_ swd_en.pdf

${ }^{55}$ Así nos lo recuerda GONZÁLEZ-PÁRAMO RODRÍGUEZ, C. en «Sector asegurador y el Derecho de la competencia. El nuevo reglamento 267/2010 de exención por categorías del sector asegurador» en la obra VV.AA. coordinada por BLANCOMORALES LIMONES, P. Estudio sobre el sector asegurador en España, pg. 276.

56 GONZÁLEZ-PÁRAMO RODRÍGUEZ, C. «Sector asegurador y el Derecho de la competencia. El nuevo reglamento 267/2010 de exención por categorías del sector asegurador»op. cit. pg. 283. 
homogeneización en el tratamiento de todos los sectores será positiva para un mercado con las especialidades que siempre han defendido las partes interesadas en el mercado de los seguros.

Aunque durante años una parte de la doctrina ${ }^{57}$ se manifestó favorable a aventurar que los reglamentos de exención por categorías «son un instrumento esencial para la seguridad jurídica del sector asegurador europeo», pues de ellos depende la armonización de una normativa que permita al sector de los seguros «desarrollar su actividad de forma idéntica en todos los Estados del Espacio Económico Europeo», las entidades aseguradoras estaban irremediablemente obligadas a prepararse para la desaparición definitiva de las exenciones en un futuro próximo. Las aseguradoras por lo tanto están obligadas a adaptar esta realidad a una mayor capacidad de autoevaluación que les permita considerar cuando sus acuerdos o prácticas quedan dentro de lo permitido por la normativa protectora del Derecho de la competencia, sometiéndose por lo tanto a las Directrices sobre aplicabilidad del artículo 101 del Tratado de Funcionamiento de la Unión Europea a los acuerdos de cooperación horizontal.

Se considera a efectos de las Directrices sobre aplicabilidad del artículo 101 del Tratado de Funcionamiento de la Unión Europea en base a su artículo 1, que una cooperación es de carácter horizontal si es objeto de un acuerdo o de prácticas concertadas concluidos entre competidores reales o potenciales. La cooperación horizontal generalmente da lugar a beneficios económicos cuando es utilizada como un medio de compartir riesgos, ahorrar costes, incrementar las inversiones, agrupar los conocimientos técnicos, aumentar la calidad y variedad del producto y lanzar más rápidamente la innovación. Igualmente una cooperación horizontal puede crear problemas de competencia cuando con ello provoca efectos negativos sobre los precios, la producción, la innovación o la diversidad y la calidad de los productos.

El objeto de estas directrices es otorgar un marco de análisis para los tipos más comunes de acuerdos de cooperación horizontal, con el fin de comprobar si son compatibles con el artículo 101 del Tratado de Funcionamiento de la Unión Europea.

${ }^{57}$ En este sentido se manifestaba DEL VALLE, M. en «El reglamento de exención: herramienta competitiva del sector asegurador europeo». Revista Española de Seguros, n. ${ }^{\circ}$ 136, 2008, pg. 610 en su análisis del derogado Reglamento 358/2003 del que decía que "todos los tipos de colaboración recogidos en el Reglamento, así como los acuerdos de liquidación de siniestros y ficheros de selección y tarificación de riesgos, suponen un claro beneficio para los consumidores y en el mercado en su conjunto». 
Cabe señalar que si el objeto de un acuerdo es restringir la competencia, esto es, que por su propia naturaleza dichos acuerdos poseen el potencial de restringir la competencia a tenor del apartado 1 del artículo 101 del TFUE, no será necesario examinar sus efectos reales o potenciales ya que en ningún momento estarán permitidos. Por otro lado, si un acuerdo de cooperación horizontal no restringe la competencia por objeto, habrá que analizar los efectos reales y potenciales para determinar si hay efectos restrictivos apreciables. Para que haya efectos restrictivos en la competencia a tenor del apartado 1 del artículo 101 del TFUE, el acuerdo deberá o debería tener un impacto adverso apreciable en al menos uno de los parámetros de competencia en el mercado - precios, producción, calidad y variedad de los productos o innovación-. Dicha evaluación de efectos restrictivos deberá hacerse teniendo en cuenta el contexto jurídico y económico real en que se daría la competencia sin el acuerdo existente.

Cuando se demuestre la presencia de una restricción de la competencia a tenor del apartado 1 del artículo 101, podrá invocarse la excepción contemplada en el apartado 3 del artículo 101. Por lo tanto, hay cuatro condiciones acumulativas que deben cumplirse para que los acuerdos de cooperación queden exentos:

a) los acuerdos deben contribuir a mejorar la producción o la distribución de los productos o a fomentar el progreso técnico o económico, es decir deben generar mejoras de eficiencia;

b) las restricciones deben ser indispensables para alcanzar esos objetivos, es decir, las mejoras de eficiencia;

c) debe reservarse a los consumidores una participación equitativa en el beneficio resultante, es decir, las mejoras de eficiencia, incluidas las cualitativas, logradas mediante las restricciones indispensables deben procurar un beneficio suficiente a los consumidores de tal modo que al menos compensen los efectos restrictivos del acuerdo; por lo tanto, no basta con que las eficiencias solo beneficien a las partes del acuerdo, a efectos de las presentes Directrices, el concepto de «consumidores» abarca los clientes, potenciales y/o reales, de las partes del acuerdo;

d) el acuerdo no debe ofrecer a las empresas la posibilidad de eliminar la competencia respecto de una parte sustancial de los productos de que se trate ${ }^{58}$.

58 Artículo 1.2.2 de Directrices sobre la aplicabilidad del artículo 101 del Tratado de Funcionamiento de la Unión Europea a los acuerdos de cooperación horizontal. 
Cuando se cumplen estos cuatro criterios, se considera que las mejoras de eficiencia que genera un acuerdo compensan la competencia generada.

Las perspectivas futuras para el buen funcionamiento del sector asegurador, pasan pues por una efectiva autoevaluación mediante la cual decidir que acuerdos serán permitidos bajo el amparo normativo comunitario ${ }^{59}$.

Si nos centramos en las dos concretas exenciones que ofrecía el REC 2010 encontramos que en la actualidad las empresas aseguradoras deben actuar de acuerdo a lo establecido en la normativa general comunitaria.

- Con respecto al intercambio de información, las directrices facilitan principios generales sobre la evaluación competitiva del intercambio de información, que se aplican a todo tipo de acuerdos de cooperación horizontal en los que se incluya el intercambio de información.

Según el artículo 2.1 de las mismas, el intercambio de información puede adoptar varias formas: los competidores pueden compartir los datos de forma directa, así como pueden compartirse los datos de forma indirecta a través de un organismo común o de un tercero o a través de los proveedores de las empresas o de los minoristas.

Ha quedado probado que el intercambio de información puede ser beneficioso para las empresas, ya que de acuerdo con lo razonado por las instituciones europea en el artículo 2.1 de las directrices horizontales, este tipo de prácticas puede solucionar problemas de asimetrías de la información, incrementando así la eficiencia de los mercados. Además, las empresas mejoran a menudo su eficiencia interna a partir de una comparación con las mejores prácticas de las demás. El intercambio de información también puede ayudar a las empresas a ahorrar costes reduciendo sus existencias, haciendo posible una entrega más rápida de los productos perecederos a los clientes o resolviendo la inestabilidad de la demanda. Asimismo, los intercambios de información pueden beneficiar directamente a los consumidores reduciendo sus costes de búsqueda y mejorando sus posibilidades de elección.

${ }^{59}$ En este sentido ALONSO SOTO, R. «Fin de la vigencia de la exención por categorías de la prohibición de los acuerdos de intercambios de información y cooperación en el sector de los seguros", Notas de Competencia, N. ${ }^{\circ} 18$, Febrero 2017, pg. 2-4. 
La cara negativa de este tipo de prácticas, puede dar lugar, en determinadas ocasiones, a efectos restrictivos de la competencia, especialmente cuando puede hacer posible que las empresas tengan conocimiento de las estrategias de mercado de sus competidores. La comunicación de información entre competidores puede constituir un acuerdo, una práctica concertada o una decisión con objeto de fijar precios o cantidades. Estos tipos de intercambios de información se considerarán carteles y, como tales, serán multados.

Fuera del ámbito de los cárteles, el intercambio de información solo se considera para restringir la competencia por objeto cuando los competidores intercambian individualmente información relativa a los precios o cantidades previstos futuros. Los intercambios del resto de tipos de información, incluidos los precios actuales, no se tratarán como restricciones por objeto y se evaluarán en términos de sus efectos restrictivos a la competencia.

- Con respecto a la segunda de las excepciones que preveía el REC 2010, es importante recordar que en el panorama nacional, el derecho español y más concretamente la Ley de ordenación, supervisión y solvencia de las entidades aseguradoras y reaseguradoras (LOSSEAR) $^{60}$ en su artículo 94.2 establece que las tarifas de primas responderán al régimen de libertad de competencia en el mercado de seguros, sin que a estos efectos, tenga el carácter de práctica restrictiva de la competencia la utilización de estadísticas comunes por parte de las entidades aseguradoras y reaseguradoras para la elaboración individual de sus tarifas de primas de riesgo, siempre y cuando dichas estadísticas se elaboren de conformidad con los reglamentos de la Unión Europea dictados para la aplicación del artículo 101.3 del Tratado de Funcionamiento de la Unión Europea, ofreciendo por lo tanto la oportunidad a las aseguradoras de continuar con su colaboración siempre y cuando se ajusten a lo establecido por la normativa europea.

Así mismo la figura del coaseguro tiene su reconocimiento y regulación en el artículo 33 de la Ley de Contrato de seguro a nivel nacional, quedando regulado el coaseguro comunitario en los artículos 12 y 103 a 105 de la LOSSEAR donde se establece el régimen normativo aplicable a estas figuras que ya no requieren una normativa específica.

${ }^{60}$ Ley 20/2015, de 14 de julio, de ordenación, supervisión y solvencia de las entidades aseguradoras y reaseguradoras, conocida como la LOSSEAR que traspone al ordenamiento jurídico español la Directiva de Solvencia II. 
Tras todo lo anteriormente expuesto, falta aún por ver si el mercado asegurador está preparado para adecuar su funcionamiento a los instrumentos de valoración y análisis otorgados por la Comisión para facilitar a las aseguradoras la posibilidad de llevar a cabo o no dichas prácticas, o para adoptar acuerdos que puedan suponer una restricción al libre juego de la competencia.

Algunos autores ya avisaron que los avances tecnológicos como la telemática y los problemas relacionados con el «Big Data», también tienen el potencial de alterar los modelos de negocio de las aseguradoras y proporcionar nuevos problemas a los reguladores. A medida que estos cambios transforman el estado del sector de los seguros, los abogados de la competencia se enfrentarán a nuevos riesgos y desafíos en los años venideros ${ }^{61}$.

Este año de observación que la Comisión está realizando sobre el sector asegurador, será fundamental para, si fuera necesario, proporcionar una orientación específica para este concreto mercado, sin duda una opción mucho más flexible y con capacidad de adaptarse a la realidad cambiante que los antiguos Reglamentos de exención.

\section{REFERENCIAS BIBLIOGRÁFICAS}

Alonso Soto, R. «La aplicación de la normativa de la competencia al seguro», Revista Española de Seguros, n. ${ }^{\circ}$ 136, 2008, pgs. 579-611.

Alonso Soto, R. «El nuevo reglamento de exención por categorías en materia de seguros». Aida-Portugal. Disponible en: http://www. aida-portugal.org/pdf/f9b31340d15447b9b4ee84e6fdab140b.pdf

Alonso Soto, R. «Fin de la vigencia de la exención por categorías de la prohibición de los acuerdos de intercambios de información y cooperación en el sector de los seguros», Notas de Competencia, n. ${ }^{\circ}$ 18, febrero de 2017, pgs. 2-4.

Bataller Grau, J.; Latorre Chiner, N.; Olavarría Iglesia, J. Derecho de los seguros privados, Marcial Pons, Madrid, 2008.

Bercovitz Rodriguez-Cano, A. Apuntes de Derecho Mercantil. Derecho Mercantil, Derecho de la Competencia y Propiedad Industrial, 16. ${ }^{\mathrm{a}}$ edición, Thomson Reuters, Cizur Menor, 2015.

${ }^{61}$ OLSEN G.; SCHWARZ, D. «Insurance services and competition law: An overview of EU and national case law», e-Competitions National Competition Laws Bulletin, n. ${ }^{\circ} 78289,2016$, pág. 4. 
Blanco-Morales Limones, P. «La aplicación del derecho europeo de la competencia a la actividad aseguradora», Revista española de seguros, n. ${ }^{\circ} 104,2000$, pgs. 731-770.

CARPagnano, M. «El private enforcement del derecho comunitario de la competencia en acción: Análisis crítico de la decisión del Tribunal de Justicia en los asuntos acumulados C295-298/04», Indret: Revista para el Análisis del Derecho, n. ${ }^{\circ}$ 1, 2007.

Del Valle, M. «El reglamento de exención: herramienta competitiva del sector asegurador europeo», Revista Española de Seguros, n. ${ }^{\circ} 136,2008$, pgs. 605-611.

Gómez del Campo, J. «El Seguro Nuclear y el Sistema de Pools», Trébol, n. ${ }^{\circ}$ 31, abril de 2004, pgs. 2-7.

GonzÁlez-PÁRAmo Rodríguez, C. «Sector asegurador y el Derecho de la competencia. El nuevo reglamento 267/2010 de exención por categorías del sector asegurador» en la obra VV.AA. coordinada por Blanco-Morales Limones, P.; Guillén Estany, M.; Domínguez FaBIÁN, I. Estudio sobre el sector asegurador en España 2010, los aspectos cualitativos de solvencia II, Fundación de estudios financieros, 2010, pgs. 275-286.

GonZÁLEZ-PÁRAMo Rodríguez, C. «El sector asegurador y el Derecho de la competencia» en la obra dirigida por Blanco Morales LimoNES, P. Estudio sobre el sector asegurador en España, Papeles de la Fundación N. ${ }^{\circ} 35$, Fundación de estudios financieros, 2010, pgs. 237-261.

Marcos Fernández, F. y Sánchez Graells, A. «Actividad aseguradora y defensa de la competencia: la exención antitrust del sector asegurador», Instituto de Ciencias del Seguro, Fundación Mapfre, 2011.

McCarthy, E. and Stefanescu, L. «The New Block Exemption Regulation for the Insurance Sector», Competition policy Newsletter, n. ${ }^{\circ} 2,2010$, pgs.6-9.

OlAVARRÍA IgLESIAS, J. «La exención por categorías de la prohibición de prácticas colusorias en el sector de los seguros: el nuevo reglamento de la Comisión Europea», Revista Española de Seguros, n. ${ }^{\circ} 145$, enero de 2011, pgs. 29-62.

Olsen, G.; Schwarz, D. «Insurance services and competition law: An overview of EU and national case law», e-Competitions National Competition Laws Bulletin, n. ${ }^{\circ}$ 78289, 2016, pgs. 1-7. 
Peñas Moyano, M.J. «La eliminación de exenciones en el sector asegurador: más autoevaluación». Revista Española de Seguros, n. ${ }^{\circ} 145$, enero de 2011, pgs. 99-117.

Peñas Moyano, M.J. «Las nuevas reglas de la competencia en el sector asegurador: acerca del Reglamento 358/2003, de 27 de febrero de 2003, relativo a la aplicación del apartado 3 del artículo 81 del Tratado a determinadas categorías de acuerdos, decisiones y prácticas concertadas en el sector de seguros», Revista española de seguros, n. ${ }^{\circ} 120,2004$, pgs. 551-590.

QUiNTÁNs EIRAS, M. ${ }^{a}$ R. «Revisión del coaseguro y del reaseguro desde el Derecho de la competencia», Revista Española de Seguros, n. ${ }^{\circ} 136$, enero de 2008, pgs. 627-641.

RYAN, S. "Commission publishes for comments a draft block exemption regulation for the insurance sector», Competition policy newsletter, n. ${ }^{\circ}$ 3, 2002, pgs. 36-38.

Veiga Copo, A. «El Derecho de Seguros en la Unión Europea», Revista Valenciana de Economía y Hacienda, n. ${ }^{\circ}$ 14, 2007, pgs. 164-187.

VV.AA. Tratado de Derecho de la competencia (BENEYTo, J.M.), Bosch, Tomo 3, 2005. 
